\title{
Il suffisso -errimo nell'italiano di oggi
}

Anna M. Thornton

PUBBLICATO: 15 GENNAIO 2021

\section{Quesito:}

Sono arrivate moltissime richieste sull'uso e sul significato di forme in -errimo.

\section{Il suffisso -errimo nell'italiano di oggi}

I 1 suffisso italiano -errimo corrisponde al latino -errimus, un suffisso che era usato per formare aggettivi esprimenti un alto grado di possesso della qualità denotata: ad esempio, pulcher 'bello', pulcherrimus 'il più bello, bellissimo'. Come si vede dalla traduzione italiana, in latino -errimus aveva un duplice valore semantico, quello normalmente denominato superlativo relativo ('il più bello') e quello denominato superlativo assoluto ('bellissimo'). Gli stessi valori aveva in latino -issimus. La scelta tra -issimus e -errimus è normalmente descritta nelle grammatiche del latino come condizionata dalla forma fonologica dell'aggettivo: si usava -errimus con aggettivi la cui radice terminava in $r$, come pulcher 'bello', pauper 'povero', piger 'pigro', ecc., e -issimus con gli altri (per es. altus 'alto' > altissimus 'altissimo'). In realtà, questa regola era prevalente ma non assoluta: le grammatiche e gli studi specialistici ci informano che già in latino sono attestate forme in -issimus da basi terminanti in $r$, per es. celerissimus in Ennio, integrissimus e miserissimus in iscrizioni. In italiano, normalmente gli aggettivi formano il cosiddetto superlativo assoluto in -issimo, anche se la radice termina in $r$ : amarissimo, carissimo, chiarissimo, durissimo, illustrissimo, leggerissimo, liberissimo, magrissimo, nerissimo, purissimo, popolarissimo, poverissimo, rarissimo, scurissimo, serissimo, severissimo, sicurissimo, tenerissimo, verissimo, per citare solo alcuni dei più frequenti di superlativi in -issimo da basi con radice terminante in $r$. Tuttavia, le grammatiche dell'italiano ricordano che sono in uso, come prestiti dotti dal latino, le seguenti forme in -errimo, elencate accanto all'aggettivo corrispondente:

$\begin{array}{lll}\text { acre } & > & \text { acerrimo } \\ \text { celebre } & > & \text { celeberrimo } \\ \text { integro } & > & \text { integerrimo } \\ \text { misero } & >\text { miserrimo } \\ \text { salubre } & >\text { saluberrimo }\end{array}$

Serianni 1988 ( $§ \mathrm{~V} 7$ oc) aggiunge inoltre che ha "ancora discreta vitalità, ma solo nella lingua letteraria, il superlativo asperrimo da aspro (cui si affianca asprissimo)".

L'elenco ci permette di rispondere già a due delle domande pervenute: di che cosa sia superlativo assoluto integerrimo (Maria Bonaria A. da Lanusei), e se "integerrimo [sia] usato anche quale superlativo assoluto di intelligente" (Manuela B. da Colle di Val d'Elsa). Come si vede, integerrimo è superlativo di integro; con intelligente condivide i fonemi iniziali e l'attacco e il nucleo della sillaba accentata (inte...gé), ma nulla più.

L'elenco appena presentato, però, da una parte non esaurisce le forme in -errimo effettivamente attestate in italiano, dall'altra non ci dice se accanto a queste forme in -errimo i primi cinque aggettivi elencati abbiano anche superlativi in -issimo, e in caso positivo se vi siano differenze di significato tra le due forme. 
Partiamo dal secondo problema, che interessa molti lettori e lettrici. La risposta non è univoca, e dovremo trattare i diversi aggettivi uno per uno.

Acrissimo è forma attestata - è registrata fin dalla terza edizione del Vocabolario degli accademici della Crusca (I69I), dove è definita "Superl. d'acro" - ma di scarsissimo uso nell'italiano contemporaneo: ad esempio, non è attestata nel corpus contenente le annate i985-200o del quotidiano "la Repubblica", dove invece acerrimo è attestato con centinaia di occorrenze. Va osservato che spesso acerrimo, etimologicamente superlativo, è trattato come se fosse un aggettivo di grado positivo: nel corpus della "Repubblica" si hanno 62 occorrenze di piu acerrim*, per lo più in contesti in cui si parla del piu acerrimo nemico, o avversario, o rivale. Il fatto che acerrimo venga usato in questa costruzione per esprimere un superlativo relativo mostra che da molti non è sentito come un superlativo assoluto, ma come un aggettivo di grado positivo, almeno se unito a questi nomi che denotano un avversario. L'analisi delle collocazioni in cui occorre acerrimo mostra che l'associazione semantica con il campo della rivalità è molto forte e decisamente prevalente per questo aggettivo, anche quando non è preceduto da piu: quindi alla domanda se "sia corretto nella nostra lingua dire limone acerrimo" (posta da Stefania M. da Roma) si può rispondere che la combinazione suona oggi quanto meno inusitata. Tuttavia tramite la ricerca in Google libri si può reperire questo brano contenuto nella Istoria e coltura delle piante che sono pe'l fiore piu ragguardevoli, e piu distinte per ornare un giardino in tutto il tempo dell'anno, con un copioso trattato degli agrumi, di Paolo Bartolomeo Clarici, pubblicata postuma a Venezia nel I726, presso Andrea Poletti:

Il Limon Selvaggio quasi rotondo è di piccola grandezza, leggiermente verrucoso, e di acerrimo sugo.

La stessa lettrice chiede se acre sia sinonimo di aspro. Almeno in relazione al sapore di un limone, i due aggettivi sembrano largamente sinonimi. Ad esempio, il Nuovo De Mauro definisce acre come "di sapore aspro, agro: il gusto acre del limone verde", e aspro come "che ha sapore acre, acidulo: uva aspra, il limone è aspro".

Infine la lettrice chiede se il superlativo assoluto di aspro sia asperrimo o asprissimo.

Asperrimo e asprissimo sono entrambi ben attestati: per esempio, hanno rispettivamente 46 e 28 occorrenze, considerando tutte le forme flesse di ciascun lemma, nel corpus "la Repubblica" I985-2000. In questo corpus, i primi collocati di asprissimo sono polemica, battaglia e lotta, e i primi di asperrimo sono scontro, battaglia, polemica, discussione elotta: come si vede, non sembra esserci differenziazione semantica tra i due superlativi.

Le forme in -issimo corrispondenti a celeberrimo, integerrimo, miserrimo e saluberrimo hanno scarsissime attestazioni: due per miserissimo, una sola per celebrissimo e integrissimo, nessuna per salubrissimo nel corpus "la Repubblica" I985-2000, a fronte di II75 occorrenze di celeberrimo, 356 di integerrimo, iı di miserrimo e due di saluberrimo, sempre considerando tutte le forme flesse del lemma. Tuttavia le forme in -issimo sono considerate esistenti e possibili dalle grammatiche: Serianni osserva che "accanto alle forme miserrimo e saluberrimo esistono i superlativi regolari miserissimo e salubrissimo". Quindi alla domanda di un lettore (Gianfranco C. da Roma) "Perchè è errato celebrissimo ed è corretto celeberrimo?" si può rispondere che celebrissimo non è veramente "errato", ma solo pochissimo usato. Dove è ben attestato nell'uso un superlativo in -errimo tratto da forme del latino, il superlativo in -issimo, formato dalla stessa base con il suffisso che forma regolarmente superlativi in italiano, non serve, perché non esprime un significato che non sia già espresso dalla forma in -errimo. Solo chi non conosca o non ricordi la forma in -errimo potrà occasionalmente formare superlativi come celebrissimo, ecc., che sono regolarissimamente formati tramite la regola produttiva per la formazione del 
superlativo assoluto in italiano contemporaneo. D'altra parte, dove porre il limite all'uso di forme in -errimo prese a prestito dal latino? Abbiamo visto che le grammatiche si limitano a ricordare i sei aggettivi sopra elencati. Ma in latino gli aggettivi che avevano superlativo in -errimo erano ben di più; alcuni, come il già ricordato pulcherrimus, non sono stati adottati in italiano perché nemmeno la loro base è sopravvissuta (oggi per esprimere il significato che in latino era espresso da pulcher diciamo bello, non pulcro, forma attestata ma di uso esclusivamente letterario, specie poetico); ma altri, legati a basi che sono di uso comune in italiano, sono a volte utilizzati. Tra questi rientra senz'altro pigerrimo, che non è di solito ricordato tra i superlativi in -errimo dalle grammatiche dell'italiano, ma è usato e oggetto di diversi quesiti pervenutici. In particolare, diverse lettrici (tra cui Francesca C. da Lanzo Torinese) dichiarano che docenti di scuola secondaria hanno censurato l'uso di pigrissimo sostenendo che il superlativo dipigro è pigerrimo ("Al liceo Parini mi hanno insegnato che pigrissimo era assolutamente da evitare!", scrive Margherita S. da Milano). In realtà, nel corpus "la Repubblica" I985-2000 si hanno due sole occorrenze di forme di pigerrimo contro 23 di forme di pigrissimo. La censura nei confronti di pigrissimo quindi probabilmente va annoverata tra quei casi in cui la tradizione diffusa nell'insegnamento scolastico sostiene l'erroneità di forme e costrutti in realtà ben attestati.

Pigerrimo e pigrissimo sono entrambi in uso, e il dubbio su quale scegliere è ben riassunto da Daniele R. da Ghezzano (PI) che così illustra il suo dilemma: "pigerrimo sembra troppo colto, pigrissimo sembra troppo banale". Le cose stanno proprio cosi. Pigerrimo sembra troppo colto perché pigerrimus non era forma di altissima frequenza neppure in latino, a differenza degli altri aggettivi in -errimus che sono alla base delle forme in -errimo di uso comune in italiano. I primi dodici superlativi in -errimus in ordine di frequenza decrescente in latino (nel corpus di testi del Packard Humanities Institute, una raccolta completa di testi latini fino al II secolo d.C.) erano pulcherrimus, acerrimus, miserrimus, celeberrimus, celerrimus, asperrimus, deterrimus, saluberrimus, uberrimus, taeterrimus, integerrimus, creberrimus (cfr. Thornton 2019). Come si vede, tra questi superlativi di alta frequenza in latino sono compresi tutti quelli il cui adattamento italiano è considerato di uso standard dalle grammatiche e ben attestato nei corpora; i superlativi latini di alta frequenza non continuati sono per lo più quelli di cui non è continuata neanche la base, come il già ricordato pulcherrimus, e deterrimus, uberrimus, creberrimus. Dunque usare pigerrimo implica conoscere (almeno un po') il latino, il che a quanto pare oggi in Italia qualifica come "troppo colti"; d'altra parte, pigrissimo è formato con la normale regola di formazione dei superlativi in italiano, che è molto produttiva (cfr. Gaeta 2003), tanto da poter apparire "banale".

È ben noto che nell'espressione dell'intensificazione i parlanti cercano espressività, non banalità. Questo spiega il reclutamento come intensificatori di parole che originariamente avevano significati diversi, ma che sembrano "meno banali" degli intensificatori più frequenti, come molto, tanto, e il suffisso -issimo. Un esempio: quando Paolo Villaggio usò l'espressione una cultura mostruosa, non intendeva certo parlare di una cultura "di estrema bruttezza", ma di una grande o grandissima cultura. L'aggettivo mostruoso viene usato qui in senso traslato per esprimere in modo "non banale" un alto grado di possesso di qualcosa. Questo desiderio di esprimere in modo non banale, a volte non disgiunto da ironia, l'alto grado di possesso di una qualità, che è normalmente espresso con forme di superlativo, sta alla base dell'uso del suffisso -errimo anche per formare il superlativo di aggettivi che normalmente lo avrebbero in -issimo (o che per lo più non si usano al superlativo), come tristerrimo e egregerrimo, segnalati in due quesiti (da parte di Annalisa F. da Taranto e Gabriele C. da Roma); nello stesso spirito c'è chi utilizza prestiti latini mai acclimatati in italiano, come magerrimo ('magrissimo') o conia addirittura pseudolatinismi quali alacerrimo, funeberrimo, luguberrimo, prosperrimo. Tra questi rientra anche un ipotetico soberrimo, che è stato già oggetto di una risposta di Vittorio Coletti. 
Una ricerca (cfr. Thornton 2019) sul corpus ItWaC Complete, che comprende testi in italiano estratti dalla rete, per oltre un miliardo e mezzo di occorrenze, ha mostrato che sono attestati oltre Ioo superlativi in -errimo, da aggettivi che non avevano il superlativo in -errimus in latino, come felicerrimo, comoderrimo, tremenderrimo, o che neppure esistevano in latino, come riccherrimo (ricco è adattamento di un prestito dal longobardo), figherrimo. Inoltre si trova -errimo unito anche a basi nominali, in forme come augurerrimi, bacerrimi, orrorerrimo, ricetterrima (lo stesso accade anche per -issimo: si pensi a partitissima, Canzonissima), o di altra categoria: sono attestati per esempio d'accorderrimo, grazierrime, presterrimo, benerrimo. In sostanza, pare che -errimo sia usato, nell'italiano contemporaneo, da parlanti che cercano consapevolmente, e quasi sempre scherzosamente, di esprimere un grado estremo di intensificazione, per il quale -issimo non è sentito come sufficiente. I due suffissi si trovano a volte anche uno di seguito all'altro, in formazioni come d'accordissimerrimo, pessimerrimissimi. Inoltre -errimo si unisce spesso a basi che gia da sole esprimerebbero la nozione di grado superlativo, come nel caso di eccellenterrimo, magnificerrimo, meraviglioserrima, ottimerrimo, splendiderrimo, stupenderrimo. Infine spesso una forma in -errimo appare in ultima posizione in climax trimembris come i seguenti (tratti dal corpus It WaC e citati in Thornton 2019):

\section{Voto al Disco: Bravi bravissimi braverrimi}

Sommamente insipido, insipidissimo, insipiderrimo, un tripudio addirittura di insipidità fu lo spettacolo di apertura della stagione del Teatro la Fenice.

Legittimo, anzi, legittimissimo, o se volete legittimerrimo, dunque, l'urlo FORZA ITALIA che prorompe dai cuori.

Insomma, -errimo appare oggi usato come mezzo stilistico per esprimere con enfasi un grado estremo di possesso di una caratteristica, soprattutto in usi informali in rete come quelli testimoniati nel corpus It WaC, e in questo uso è spesso unito a basi che non corrispondono a basi possibili del suo antenato latino -errimus. In usi più formali, come quelli attestati dal corpus "la Repubblica" I985-2000, -errimo si trova invece quasi solo con aggettivi che avevano un superlativo in -errimus in latino ed erano in latino di alta frequenza.

\section{Nota bibliografica:}

- Livio Gaeta, Produttivitá morfologica verificata su corpora: il suffisso -issimo, in I nuovi media come strumenti per la ricerca linguistica, a cura di Franz Rainer e Achim Stein, Frankfurt am Main/Berlin/Bern/Bruxelles/New York/Oxford/Wien, Peter Lang, 2003, pp. 43-6o.

- Anna M.Thornton, Sulle forme in -errimo nellitaliano contemporaneo, "Studi di grammatica italiana" XXXVIII, 2019, pp. 301-332.

\section{Cita come:}

Anna M. Thornton, I/ suffisso errimo nell'italiano di oggi , "Italiano digitale", 2021, XVI, 2021/1 (gennaio-marzo)

DOI: $10.35948 / 2532-9006 / 2021.5459$

Copyright 2021 Accademia della Crusca

Pubblicato con licenza creative commons CC BY-NC-ND 